\title{
Efficient yeast cell-surface display of exo- and endo-cellulase using the SED1 anchoring region and its original promoter
}

\author{
Kentaro Inokuma ${ }^{1}$, Tomohisa Hasunuma ${ }^{2}$ and Akihiko Kondo ${ }^{1,3,4^{*}}$
}

\begin{abstract}
Background: The recombinant yeast strains displaying the heterologous cellulolytic enzymes on the cell surface using the glycosylphosphatidylinositol (GPI) anchoring system are considered promising biocatalysts for direct conversion of lignocellulosic materials to ethanol. However, the cellulolytic activities of the conventional cellulase-displaying yeast strains are insufficient for the hydrolysis of cellulose. In this study, we constructed novel gene cassettes for the efficient cellulose utilization by cellulase-displaying yeast strains.
\end{abstract}

Results: The novel gene cassettes for the cell-surface display of Aspergillus aculeatus $\beta$-glucosidase (BGL1) and Trichoderma reeseii endoglucanase II (EGII) were constructed using the promoter and the GPI anchoring region derived from Saccharomyces cerevisiae SED1. The gene cassettes were integrated into the S. cerevisiae genome, then the $\beta$-glucosidase activity of these recombinant strains was evaluated. We revealed that simultaneous utilization of the SED1 promoter and Sed1 anchoring domain in a gene cassette enabled highly-efficient enzyme integration into the cell wall. The $\beta$-glucosidase activity of recombinant yeast cells transduced with the novel gene cassette was 8.4-fold higher than that of a conventional strain. The novel EGIl-displaying strain also achieved 106-fold higher hydrolysis activity against the water-insoluble cellulose than a conventional strain. Furthermore, direct ethanol production from hydrothermally processed rice straw was improved by the display of $T$. reeseii EGll using the novel gene cassette.

Conclusions: We have developed novel gene cassettes for the efficient cell-surface display of exo- and endo-type cellulolytic enzymes. The results suggest that this gene cassette has the wide applicability for cell-surface display and that cellulase-displaying yeasts have significant potential for cost-effective bioethanol production from lignocellulosic biomass.

Keywords: Saccharomyces cerevisiae, Cell-surface display, ß-glucosidase, Endoglucanase, Bioethanol production, Lignocellulosic biomass

\section{Background}

The search for practical petroleum substitutes from renewable resources has become a global priority as atmospheric carbon dioxide levels continue to rise. Lignocellulosic materials such as sugar cane bagasse, corn stover, rice straw, grasses, wood chips and other agricultural waste contain large amounts of polysaccharides such as cellulose and

\footnotetext{
* Correspondence: akondo@kobe-u.ac.jp

'Department of Chemical Science and Engineering, Graduate School of Engineering, Kobe University, 1-1 Rokkodai, Nada, Kobe 657-8501, Japan ${ }^{3}$ Biomass Engineering Program, RIKEN, 1-7-22 Suehiro-cho, Tsurumi-ku, Yokohama, Kanagawa 230-0045, Japan

Full list of author information is available at the end of the article
}

hemicellulose. These polysaccharides are one of the most abundant renewable resources in nature, and are attracting much attention as feedstocks for second-generation bioethanol production [1]. However, due to the recalcitrance of lignocellulosic materials, the pre-treatment requiring a large amount of energy and the large quantities of multiple enzymes are necessary for the complete hydrolysis of these polysaccharides to fermentable sugars [2-5]. The cost of the bioconversion of these materials to ethanol is much higher than that of conventional sugar sources such as corn starch and cane juice [1]. In particular, the cost of the enzymes is one of the biggest obstacles for commercially viable production of bioethanol from lignocellulosic 
materials. Therefore, consolidated bioprocessing (CBP), which combines enzyme production, saccharification of polysaccharides, and fermentation into a single process, has a great potential for the cost-effective production of ethanol from lignocellulosic materials [6-8]. To date, however, no natural microorganisms with the capability for efficient enzyme production, lignocellulose saccharification, and ethanol production have been identified.

Cellulose, a polymer of $\beta$-(1 to 4$)$ linked glucose residues, is the major component of lignocellulosic materials (40 to $60 \%$ by total dry weight) [9]. Saccharomyces cerevisiae (S. cerevisiae) is the most commonly used microorganism for industrial ethanol production. Compared to bacteria, it has more rapid sugar consumption, provides a higher ethanol yield from glucose, and has higher resistance to ethanol and other compounds present in lignocellulosic hydrolysates $[10,11]$. However, native $S$. cerevisiae is not a candidate microorganism for the CBP of cellulose because it lacks the enzymes required for the hydrolyzation of cellulose into glucose. To address this problem, the heterologous expression and secretion of cellulolytic enzymes, including endoglucanase (EG), cellobiohydrolase $(\mathrm{CBH})$ and $\beta$-glucosidase (BGL) from yeasts and other microorganisms, have been pursued over the last two decades [12]. On the other hand, efficient whole-cell biocatalysts for simultaneous saccharification and fermentation have also been constructed by displaying cellulolytic enzymes on the cell surface using the glycosylphosphatidylinositol (GPI) anchoring system [13-18]. The cell-surface engineering system has several industrial advantages over the enzyme secretion system. In this system, yeast is transformed by introducing fusion genes coding cellulolytic enzymes and the anchoring domain of GPI protein. The fused proteins self-immobilize into the yeast cell wall, so the activities of the enzymes are retained as long as the yeast continue to grow [19]. As the separation of the biocatalyst from the products is easy, reutilization of the yeast cells also enables re-use of the active enzymes displayed on the cell surface, without the need for the cells to reproduce. This approach would reduce the cost of yeast propagation as well as the cost of enzyme addition [20]. Furthermore, Matano et al. [21] recently reported a cell-surface display system that alleviated the adsorption of free cellulases onto the crystalline cellulose and improved the hydrolysis of cellulose and ethanol production.

In previous studies, the C-terminal domain of $\alpha$-agglutinin (Sag1), which is a GPI protein in S. cerevisiae, has been mainly used as an anchoring domain in fusion proteins. The genes encoding these fusion proteins have been expressed by constitutive promoters such as TDH3 and PGK1 promoters. However, the cellulolytic activities on the cell surface provided using conventional gene cassettes are insufficient for the efficient CBP of cellulose. There is therefore, urgent need for improvements in yeast cellsurface display cassettes.

In this study, we constructed the novel gene cassettes for the cell-surface display of cellulolytic enzymes that incorporated the promoter and the GPI anchoring region derived from $S$. cerevisiae SED1, which encodes a major stress-induced structural GPI protein [22]. The gene cassettes for the cell-surface display of Aspergillus aculeatus $\beta$-glucosidase (BGL1) were integrated into the HIS3 locus of the S. cerevisiae genome, then the BGL activity of these recombinant strains was evaluated. The results demonstrated that the simultaneous utilization of the SED1 promoter and the anchoring region significantly improved BGL activity on the cell surface. In addition, we also constructed a recombinant yeast strain displaying the Trichoderma reeseii endoglucanase II (EGII) using this novel gene cassette. Hydrolysis activity against the water-insoluble cellulose of this strain also increased significantly. Furthermore, the ethanol productivity from hydrothermally processed rice straw was clearly improved by using this EGII-displaying strain.

\section{Results}

\section{Construction of yeast strains}

A haploid yeast strain, S. cerevisiae BY4741 was used as the host strain in this study. Figure 1 shows the four types of gene cassettes constructed for cell-surface display of cellulolytic enzymes in this study. The TA

$$
\begin{aligned}
& \begin{array}{l|l|l|l|l|l|}
\text { TA } & T D H 3 \text { promoter } & \text { S. S. } & B G L 1 \text { or } E G I I \text { gene } & \text { SAG1 anchor } & \text { SAG1 terminator } \\
\text { TS } T D H 3 \text { promoter } & \text { S. S. } & B G L 1 \text { or } E G I \text { gene } & S E D 1 \text { anhcor } & S A G 1 \text { terminator } \\
\hline
\end{array}
\end{aligned}
$$

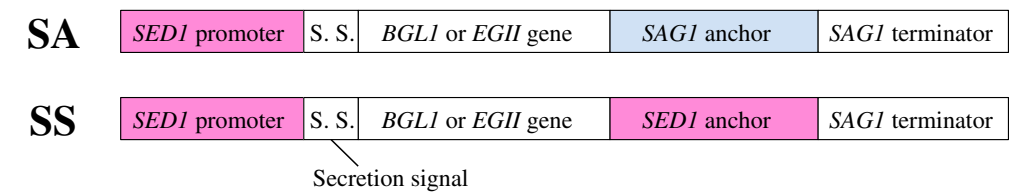

Figure 1 Construction of novel gene cassettes for the yeast cell-surface display of cellulolytic enzymes. All cassettes have the secretion signal sequence of the Rhizopus. oryzae glucoamylase gene and SAG1 terminator. 
cassette is a conventional cassette containing the $S$. cerevisiae TDH3 promoter and the 3'-half of S. cerevisiae SAG1 as a GPI anchoring region. The novel gene cassettes are hereinafter referred to as TS (TDH3 promoter and $S$. cerevisiae SED1 anchoring region), SA (SED1 promoter and SAG1 anchoring region), and SS cassettes (SED1 promoter and SED1 anchoring region). All cassettes have the secretion signal sequence of the Rhizopus oryzae glucoamylase gene and SAG1 terminator. The plasmids with these cassettes (Table 1) were integrated into the HIS3 locus of the chromosomal DNA by homologous recombination. The constructed strains listed in Table 2 were used for the experiments described below.

\section{Transcriptional levels of BGL1 and BGL activities of recombinant yeast strains}

$\beta$-glucosidase (BGL) cleaves cello-oligosaccharides from the non-reducing end to give glucose in the last step of enzymatic cellulose degradation. This enzyme is required for the complete hydrolysis of cellulose. Recombinant strains harboring gene cassettes for cell-surface display of $A$. aculeatus BGL1 (BY-BG-TA, TS, SA and SS strains in Table 2) were cultivated aerobically for $96 \mathrm{~h}$. The culture broth was sampled every $24 \mathrm{~h}$, and the transcriptional level of BGL1 and BGL activity in the cell was investigated as described in Methods. Throughout the cultivation period, the transcriptional levels of BGL1 controlled by the SED1 promoter (BY-BG-SA and SS) were far higher than that of the $T D H 3$ promoter (BY-BG-TA and TS) (Figure 2A). Whereas BGL1 expression controlled by the $T D H 3$ promoter gradually increased during $72 \mathrm{~h}$ of cultivation, the expression under the control of the SED1 promoter rose dramatically and rapidly during the first $48 \mathrm{~h}$ of cultivation.

BGL activity of BY-BG-TA ( $28 \pm 3 \mathrm{U} / \mathrm{g}$ dry cells at $48 \mathrm{~h}$ ) was slightly improved by the replacement of the GPI anchoring domain by the Sed1 protein (BY-BG-TS, $32 \pm$ $6 \mathrm{U} / \mathrm{g}$ dry cell at $48 \mathrm{~h}$ ). Promoter replacement by the SED1 promoter resulted in significantly improved BGL activity (BY-BG-SA). BGL activity of BY-SBGA reached $99 \pm 10 \mathrm{U} / \mathrm{g}$ dry cells after $48 \mathrm{~h}$ cultivation (Figure 2B). Furthermore, simultaneous utilization of the SED1 promoter and Sed1 anchoring domain (BY-BG-SS) achieved more than double the BGL activity of BY-BG-SA (235 \pm $28 \mathrm{U} / \mathrm{g}$ dry cells at $48 \mathrm{~h}$ ) to approximately 8.4-fold higher than that of the conventional strain, BY-BG-TA. These results suggested a synergetic effect of the SED1 promoter and Sed1 anchoring domain for the cellsurface display of enzymes. In all strains investigated, most of the BGL activity was immobilized on the yeast cell surface and only a small amount of activity was detected in the culture supernatants throughout the cultivation (Figure 2C). No significant difference was observed in the cell growth of the various strains (data not shown).

\section{Kinetic parameters of BGL displayed on the cell surface}

To characterize the BGL activities of BY-BG-SA and SS, their kinetic parameters were determined. For the determination of the $K_{m}$ and $V_{\max }$, BGL activity was measured between 0.02 and $0.5 \mathrm{mM} p$-nitrophenyl- $\beta$-Dglucopyranoside ( $p \mathrm{NPG}$ ) (Figure 3 ). Kinetic parameters were calculated by weighted non-linear least-squares

Table 1 Characteristics of the integrative plasmids used in this study

\begin{tabular}{|c|c|c|}
\hline Plasmids & Relevant genotype & Source/references \\
\hline pRS403 & HIS3 Integrative vector without display cassette & Agilent Technologies \\
\hline p $\delta U-P G A G E G$ & URA3 $\delta$-Integrative vector with cell-surface display cassette of T. reesei EGIl & {$[26]$} \\
\hline plBG13 & $\begin{array}{c}\text { HIS3 Integrative vector of cell-surface display cassette with TDH3 promoter, } \\
\text { SAG1 anchoring region and SAG1 terminator }\end{array}$ & [43] \\
\hline plBG13S & $\begin{array}{c}\text { HIS3 Integrative vector of cell-surface display cassette with TDH3 promoter, } \\
\text { SED1 anchoring region and SAG1 terminator }\end{array}$ & This study \\
\hline pISBG13 & $\begin{array}{c}\text { HIS3 Integrative vector of cell-surface display cassette with SED1 promoter, } \\
\text { SAG1 anchoring region and SAG1 terminator }\end{array}$ & This study \\
\hline plBG-TA & HIS3 TDH3p-A. aculeatus BGL1-SAG1 ${ }_{A}-S A G 1_{T}$ & This study \\
\hline plBG-TS & HIS3 TDH3 ${ }_{p}-$ A. aculeatus BGL1-SED1 ${ }_{A}-S A G 1_{T}$ & This study \\
\hline pIBG-SA & HIS3 SED1 ${ }_{p}-$ A. aculeatus BGL1-SAG1 ${ }_{A}-S A G 1_{T}$ & This study \\
\hline plBG-SS & HIS3 SED1 $1_{P}-A$. aculeatus BGL1-SED1 ${ }_{A}-S A G 1_{T}$ & This study \\
\hline plEG-TA & HIS3 TDH3 ${ }^{-}$T. reesei EGII $-S A G 1_{A}-S A G 1_{T}$ & This study \\
\hline plEG-TS & HIS3 TDH3 - -T. reesei EGII $-S E D 1_{A}-S A G 1_{T}$ & This study \\
\hline plEG-SA & HIS3 SED1 $-T$. reesei $E G \| I--S A G 1_{A}-S A G 1_{T}$ & This study \\
\hline plEG-SS & HIS3 SED1 $1_{p}-T$. reesei $E G I I-S E D 1_{A}-S A G 1_{T}$ & This study \\
\hline
\end{tabular}

T. reesei, Trichoderma reesei; A. aculeatus, Aspergillus aculeatus. 
Table 2 Characteristics of the yeast strains used in this study

\begin{tabular}{ccc}
\hline Strains & Relevant genotype & Source \\
\hline BY4741 & MATa his3 $\triangle 1$ leu2 $\triangle 0$ met15 $\triangle 0$ ura3 $\triangle 0$ & Life Technologies \\
BY-403 & BY4741/pRS403 & This study \\
BY-BG-TA & BY4741/plBG-TA & This study \\
BY-BG-TS & BY4741/plBG-TS & This study \\
BY-BG-SA & BY4741/plBG-SA & This study \\
BY-BG-SS & BY4741/plBG-SS & This study \\
BY-EG-TA & BY4741/plEG-TA & This study \\
BY-EG-TS & BY4741/plEG-TS & This study \\
BY-EG-SA & BY4741/plEG-SA & This study \\
BY-EG-SS & BY4741/plEG-SS & This study \\
\hline
\end{tabular}

analysis of the raw data to fit to the Michaelis-Menten function [23]. The $V_{\max }$ values of BGL activity for $p$ NPG cleavage in BY-BG-SA and SS strains were 148 and 326 $\mathrm{U} / \mathrm{g}$ dry cells, respectively (Figure 3 ). In contrast, no significant difference was observed in the $K_{m}$ value of these strains (0.181 and $0.198 \mathrm{mM}$, respectively). Generally in enzyme kinetics, such a change in kinetic parameters is due to changes in the amount of active enzyme per unit of protein weight. As the measurement unit of BGL activity is defined as per unit cell-weight (U/g dry cells) in this study, these results suggest that the amount of active enzyme per unit cell-weight differs substantially between BY-BG-SA and SS, and it accounts for the difference in BGL activity.

\section{Cell-surface display of $T$. reeseii EGII}

Multiple enzymes, including endo-type cellulases, are necessary for the hydrolysis of cellulose [5]. EG, which cleaves glycosidic linkages randomly along cellulose chains, is also one of the enzymes required for the complete hydrolysis of cellulose, as is BGL. To test the applicability of the SED1 promoter and the anchoring region for cell-surface display of an endo-type cellulase, T. reesei EGII-displaying yeast strains BY-EG-TA, TS, SA and SS were constructed (Table 2). These strains were cultured under aerobic conditions at $30^{\circ} \mathrm{C}$ for $48 \mathrm{~h}$, then the enzyme activity of the cells against water-insoluble substrates was evaluated using azurine cross-linked hydroxyethylcellulose (AZCL-HE-Cellulose). The results are shown in Table 3. The SED1 promoter had a positive effect on the hydrolysis of AZCL-HE-Cellulose, as it had for the BGL activity (Figure 2B). The AZCL-HECellulose hydrolysis activity of BY-EG-TA was significantly improved by using the Sed1 anchoring domain (BY-EG-TS), although there was only a small increase in BGL activity between BY-BG-TA and TS (Figure 2B). The hydrolysis activity of BY-EG-TS was 72-fold higher than that of the conventional strain BY-EG-TA. Furthermore,

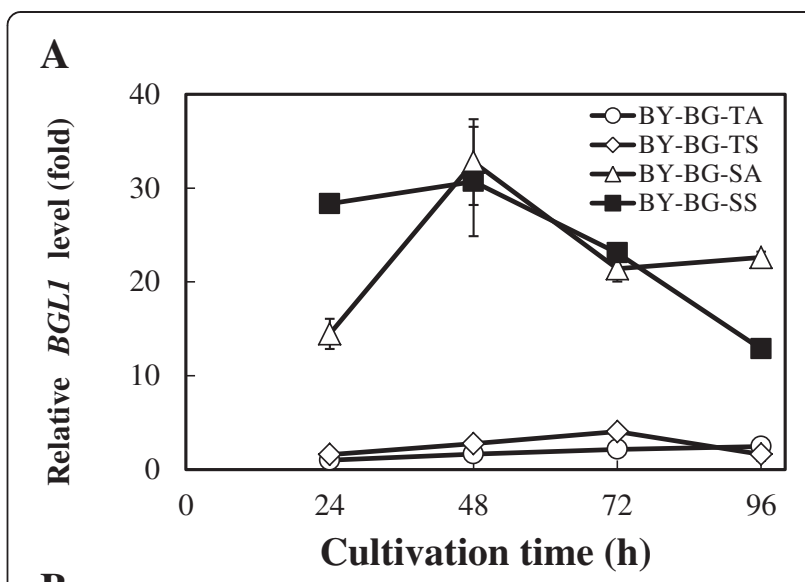

B

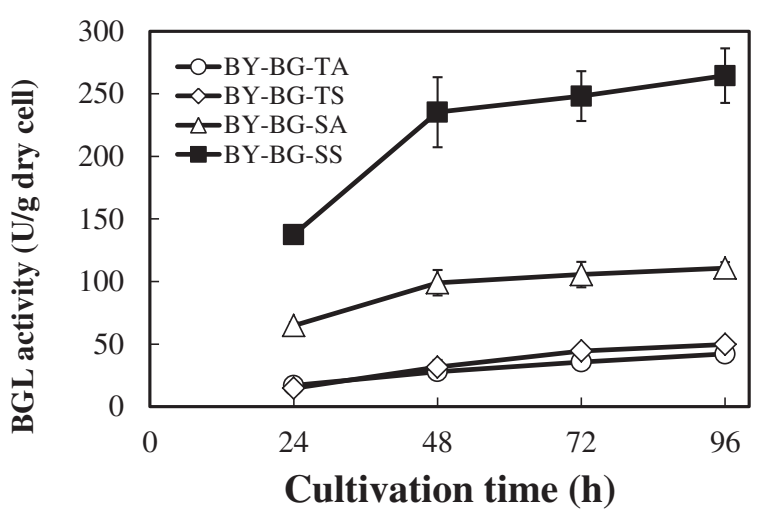

C

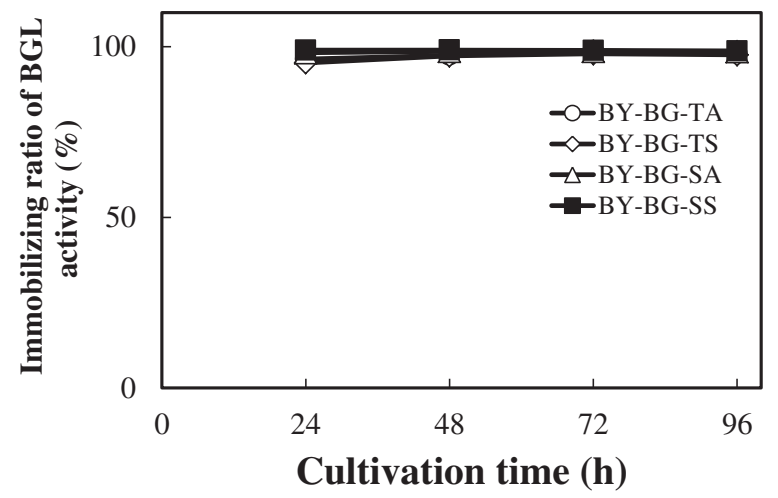

Figure 2 Characterization of $\beta$-glucosidase (BGL)-displaying yeast strains. (A) Time course of transcriptional levels of BGL1. The relative $B G L 1$ level of each sample is shown as a fold change in the mRNA level from the average of BY-BG-TA in $24 \mathrm{~h}$. (B) Time course of $B G L$ activities. (C) Immobilizing ratios of BGL activity. The immobilizing ratio was calculated by dividing the activity of the cell by the total activity of the culture medium, including the culture supernatant. Error bars indicate the standard deviations of three independent experiments.

BY-EG-SS showed 106-fold higher activity against AZCLHE-Cellulose than BY-EG-TA. Although a measurement of AZCL-HE-Cellulose hydrolysis activity does not provide absolute values of EG activity [24], these results indicated that the gene cassettes carrying the Sed1 anchoring domain 


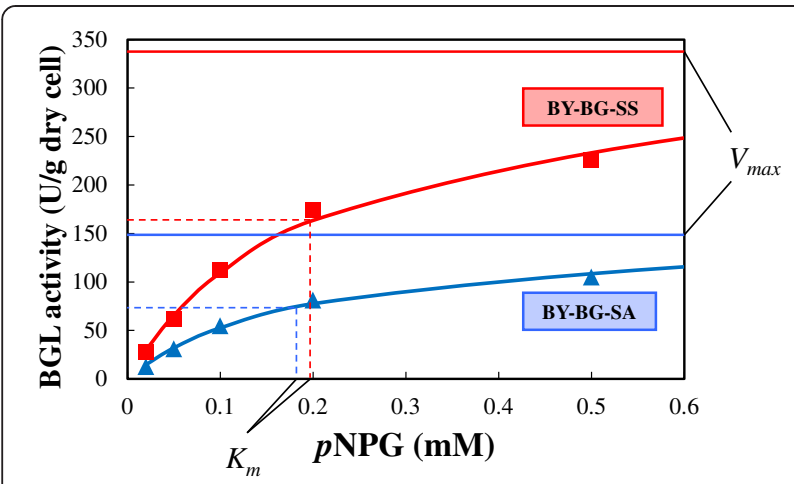

Figure 3 Kinetic parameters of the $\beta$-glucosidase (BGL)-displaying strains. After $48 \mathrm{~h}$ cultivation, BGL activity of BY-BG-SA and SS cells was measured between 0.02 and $0.5 \mathrm{mM}$ p-nitrophenyl- $\beta$-D-glucopyranoside (pNPG) at $\mathrm{pH} 5.0$ and $30^{\circ} \mathrm{C} . K_{m}$ and $V_{\max }$ values were calculated by weighted non-linear least-squares analysis of the raw data [23]. SA, SED1 promoter and SAG1 anchoring region; SS, SED1 promoter and SED1 anchoring region.

are more favorable for the cell-surface display of endo-type cellulases than those with the Sag1 anchoring domain.

\section{Direct ethanol production from hydrothermally processed rice straw}

In order to evaluate the effects of the cell-surface display of EGII on the hydrolysis of lignocellulosic biomass, direct ethanol production from hydrothermally processed rice straw was performed using the cells of EGIIdisplaying strains BY-EG-TA and SS. One filter paper unit (FPU)/g-biomass of commercial cellulase cocktail (Cellic CTec2) was added to the fermentation mixture to supply other cellulolytic enzymes. Fermentation using the BY-403 strain, which was transformed with an empty vector (pRS403), was also carried out as a control. As shown in Figure 4, no significant difference was observed in ethanol productivity from the pretreated rice straw between BY-EG-TA and the control strain (11.1 \pm 0.6 and $11.1 \pm 0.9 \mathrm{~g} / \mathrm{L}$ of ethanol after $96 \mathrm{~h}$, respectively). On the other hand, the ethanol productivity of this fermentation was clearly improved by using BY-EG-SS strain $(13.6 \pm 0.5 \mathrm{~g} / \mathrm{L}$ of ethanol after $96 \mathrm{~h})$.

Table 3 AZCL-HE-Cellulose hydrolysis activity of EGIIdisplaying yeast strains

\begin{tabular}{lc}
\hline Strains & EG activities $\left(\mathbf{A}_{\mathbf{5 9 0}}\right)^{\mathbf{a}}$ \\
\hline BY-EG-TA & $0.030 \pm 0.004$ \\
BY-EG-TS & $2.157 \pm 0.361$ \\
BY-EG-SA & $0.053 \pm 0.015$ \\
BY-EG-SS & $3.188 \pm 0.133$
\end{tabular}

${ }^{a}$ Enzyme activity was evaluated based on the absorbance at wavelength of $590 \mathrm{~nm}$ in a $1 \mathrm{~cm}$ optical path length cuvette $\left(A_{590}\right)$ of the blue dye released into the supernatant after incubation for $4 \mathrm{~h}$ at $38^{\circ} \mathrm{C}$. The averages for three independent experiments are shown with their standard deviations.

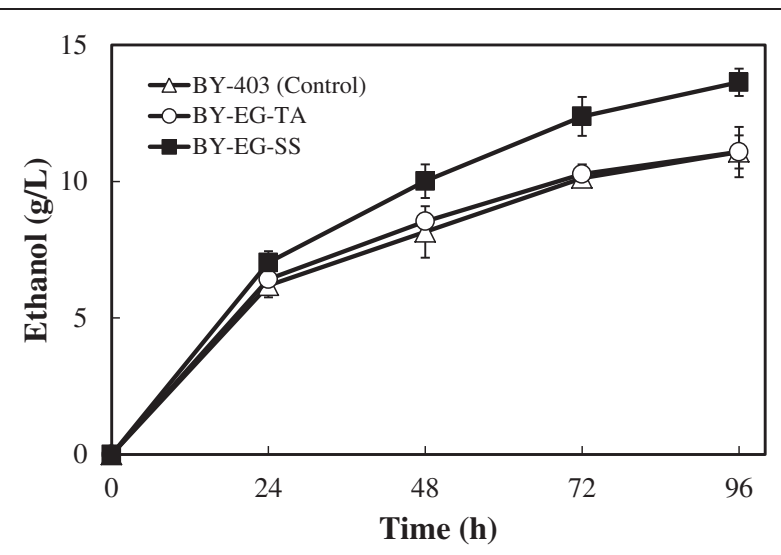

Figure 4 Time course of direct ethanol production from $100 \mathrm{~g}$ dry weight/L of hydrothermally processed rice straw. Error bars indicate the standard deviations of three independent experiments.

\section{Discussion}

In this study, we developed novel gene cassettes for the efficient cell-surface display of cellulolytic enzymes using the $S$. cerevisiae SED1 promoter and anchoring region (Figure 1). BGL-displaying strains using the novel gene cassettes (BY-BG-TS, SA and SS) achieved higher BGL activity on the cell surface than a conventional strain (BY-BG-TA) harboring a cassette carrying the TDH3 promoter and the SAG1 anchoring region (Figure 2B). The BY-BG-SS strain, which had a gene cassette with the $S$. cerevisiae SED1 promoter and its anchoring region (SS cassette), achieved the highest BGL activity (Figure 2B). Comparison of GPI anchoring domains for effective cell-surface display using the inducible GAL7 promoter has been reported [25]. However, there have been no reports on the effect of the combinations of a promoter and an anchoring domain for enzyme cellsurface display. To our knowledge, this is the first report on the synergetic effect of a GPI anchoring domain and its original promoter for the cell-surface display.

Previously, the activity of heterologous enzymes displayed on a yeast cell surface was improved by increasing the copy number of the gene cassettes, for example by using the multi-copy genome integration method ( $\delta$-integration) [26]. However, the enzyme activity of recombinant strains constructed by the $\delta$-integration method is relatively unstable, due to the random genome integration of gene cassettes. Yamada et al. [26] constructed a recombinant $S$. cerevisiae strain, MT8-1/ $\delta B E C$, by $\delta$-integration. MT8-1/8BEC had six copies of a gene cassette for BGL display using the $P G K 1$ promoter and the Sag1 anchoring domain. MT8-1/SBEC achieved a BGL activity of $15 \mathrm{U} / \mathrm{g}$ wet cells, which is comparable to approximately $100 \mathrm{U} / g$ dry cells after $72 \mathrm{~h}$ cultivation. On the other hand, the BGL activity of BY-BG-SS reached $235 \pm 28 \mathrm{U} / \mathrm{g}$ dry cells after only $48 \mathrm{~h}$ cultivation, even 
though it carried only a single gene cassette for BGL display. Although these strains cannot be compared directly, because MT8-1 and BY4741 strains have different genetic backgrounds, this novel gene cassette will thus allow the more stable construction of a heterologous enzymedisplaying yeast strain with high activity. The BGL activity of BY-BG-SS ( $235 \pm 28 \mathrm{U} / \mathrm{g}$ dry cells after $48 \mathrm{~h}$ ) is close to the specific activity of purified $A$. aculeatus BGL1 (280.7 $\mathrm{U} / \mathrm{g}$ protein) at $\mathrm{pH} 5.0$ and $30^{\circ} \mathrm{C}$ [27]. The $K_{m}$ value of the BGL on the BY-BG-SS strain for $p$ NPG $(0.198 \mathrm{mM})$ is lower than that of purified A. aculeatus BGL1 (0.23 mM) [27]. Furthermore, SS cassettes also have a significant effect on the cell-surface display of $T$. reeseii EGII, which is an endo-type cellulolytic enzyme. Hydrolysis activity of BYEG-SS against the water-insoluble substrate, AZCL-HECellulose, was 106-fold higher than that of the conventional strain, BY-EG-TA (Table 3). These results suggest that recombinant yeast cells displaying cellulolytic enzymes using SS cassettes are highly promising biocatalysts for efficient ethanol production from cellulosic materials.

It has been reported that the SED1 expression level increases during the stationary phase [22]. As shown in Figure 2A, the expression level of the BGL1 controlled by $S E D 1$ promoter rose dramatically and rapidly after 48 $\mathrm{h}$ cultivation. The BGL1 activity of BY-BG-SA and SS was 3.5- and 7.4-fold higher than that of BY-BG-TA and TS after $48 \mathrm{~h}$, respectively (Figure $2 \mathrm{~B}$ ). The high expression level of BGL1 controlled by the SED1 promoter is likely one of the causes for the high BGL activity of the BY-BG-SA and SS strains. On the other hand, it was previously reported that the mRNA level of SED1 varies periodically during the cell cycle and that the peak of SED1 expression is M phase, whereas no apparent peak was observed in $T D H 3$ expression during the cell cycle [28]. In the cell cycle of S. cerevisiae, the synthesis of the new cell wall is activated in the $M$ phase. The expression of many genes involved in the synthesis of cell-wall components and their delivery also increases in this phase [28], suggesting that the $M$ phase is the most suitable for efficient cell wall integration of GPI proteins. Although further investigation using other constitutive promoters such as PGK1 promoter is needed, the cell cycle-regulated expression controlled by the SED1 promoter might be also one of the causes of high BGL activity of the BY-BG-SA and SS strains (Figure 2B).

The result shown in Figure 3 suggests that the amount of BGL1 immobilized in the cell wall varies depending on the C-terminal anchoring domain, and accounts for the difference in BGL activity between the BY-BG-SA and SS strains. It has been estimated that $S$. cerevisiae cell contains the 60 GPI proteins, and most of them can be integrated into the cell wall by a covalent attachment to the $\beta$-(1 to 6) glucan chains in the glucan layer [29]. In recombinant yeasts, heterologous GPI-anchoring fusion proteins must compete with the native GPI proteins for the limited protein incorporation capacity of the glucan layer [25]. Therefore, it is possible that the integration efficiency of GPI-anchoring enzymes strongly affects the population and activity of the enzymes in the cell wall [25]. It was recently reported that different GPI proteins exhibit different transportation and integration efficiencies into the cell wall [30-34]. Analysis of a large panel of biochemically verified GPI proteins suggested that specific residues in the upstream region of the $\omega$-site, which is the cleavage/attachment site of the C-terminal GPI-anchoring signal recognized by a GPI transamidase, seem to favor cell wall localization [32-34]. Hamada et al. [33] reported that the amino acid sequence in the upstream region of $\omega$-site differed substantially between Sed1 and Sag1, and that reporter protein fused with the Sed1 anchoring domain had higher cell-wall integration efficiency than protein fused with the Sag1 anchoring domain. The results shown in Figures 2 and 3 are in good agreement with these reports.

Another possible cause for the high BGL activity of the BY-BG-SS strain is the specific synergy of the SED1 promoter with the Sed1 anchoring domain in SS cassette. We constructed novel gene cassettes for BGL1 display with the $S$. cerevisiae CWP2 promoter and/or its anchoring region based on the SS cassette (Additional file 1). Cwp2 is a major GPI protein in S. cerevisiae, as is Sed1 [35]. The mRNA peak level of CWP2 during the cell cycle occurs during the G2 phase [28]. BGLdisplaying strains harboring these cassettes (BY-BG-SC, $\mathrm{CS}$ and $\mathrm{CC}$ ) were constructed and their BGL activity was evaluated (Additional file 2). BY-BG-CC achieved the highest BGL activity among these strains (148 \pm 1 $\mathrm{U} / \mathrm{g}$ dry cells after $48 \mathrm{~h}$ ), whereas BY-BG-SC exhibited less than half the BGL activity of BY-BG-CC $(71 \pm 2 \mathrm{U} / \mathrm{g}$ dry cells after $48 \mathrm{~h}$ ) (Additional file 2). These results suggest that each gene encoding GPI anchoring protein is expressed most efficiently at different phases during the cell cycle, as regulated by its original promoter in S. cerevisiae cells. After the attachment of the GPI anchor in the endoplasmic reticulum (ER), GPI proteins leave the ER in COPII-coated vesicles and travel via the Golgi to the plasma membrane [36]. During this intracellular transportation, each GPI protein and its GPI anchors are subject to a variety of modifications [29]. Although many aspects of the intracellular transportation and modification systems for each GPI protein remain unclear [29], cell cycle-regulated expression of a gene encoding GPI protein under the control of its original promoter might favor efficient intracellular transportation and modification. Further validation for other GPI proteins and their promoters is needed to prove this synergetic effect. Regardless, the simultaneous utilization of an anchoring domain of GPI protein and its original promoter is a 
good option for constructing gene cassettes providing efficient cell-surface display.

The cell wall of yeast is made of a thick microfibrillar array of $\beta$-(1 to 3$)$ glucan and $\beta$-(1 to 6$)$ glucan chains [37]. It is possible that a part of GPI anchoring enzymes expose their catalytic domain to external surface of the cell wall while the remainder is completely embedded in the glucan layer [25]. Small substrates such as cellobiose are accessible to all integrated enzymes because these substrates penetrate the cell wall. In contrast, large substrates such as cellulose can only access enzymes exposed on the external surface. Therefore, the hydrolysis of highly polymerized substrates by enzymes integrated into the yeast cell wall is more difficult than hydrolysis of small substrates [25,38]. In this study, we constructed several T. reeseii EGII-displaying strains (BY-EG-TA, TS, SA and SS) and evaluated the enzyme activity of these cells using AZCL-HE-Cellulose as the water-insoluble substrate. Whereas BY-EG-TA and SA had very little activity against AZCL-HE-Cellulose, BY-EG-TS and SS showed significant activity (Table 3 ). This result suggests that the Sed1 anchoring domain provides significant advantages over the Sag1 anchoring domain not only in the efficiency of cell wall integration, but also in localizing the enzyme at the external surface of the cell wall. We constructed the novel gene cassette for EGII display with the S. cerevisiae CWP2 promoter and its anchoring region (Additional file 1). The EG-displaying strain harboring this cassette (BY-EG-CC) was constructed and the hydrolysis activity against AZCL-HE-Cellulose was evaluated. The hydrolysis activity of BY-EG-CC $\left(\mathrm{A}_{590}=\right.$ $0.021 \pm 0.001)$ was lower than BY-EG-TA $(0.030 \pm$ 0.004), even though BGL-displaying strain using the CC cassette (BY-BG-CC) achieved high BGL activity (Additional file 2). This result also suggests that the anchoring domain has significant effect on the localization of enzymes in the cell wall. On the other hand, the possibility of the effect of the steric hindrance by different anchoring domains fused with EGII cannot be excluded. Although the full length of Sed1 was used as the Sed1 anchoring domain in this study, it has been reported that C-terminal-229 amino acids of Sed1 can be used as an anchoring domain [25]. It cannot be denied that the extra $\mathrm{N}$-terminal sequence of Sed1 could function as a linker, causing the positive effect of the substrate accessibility of EGII. Investigation of the surface accessibility of the displayed enzymes by other experiments such as immunofluorescence labeling [39] would be necessary for more understanding about the localization of Sed1 in the cell wall.

SED1 is a major stress-induced gene in S. cerevisiae, and the expression level increases during stressful industrial fermentation such as sake brewing $[22,40]$. Hasunuma and Kondo [1] suggested that the environment of an economically feasible CBP using cellulase-displaying yeast strains must also be stressful (highly concentrated yeast cells, high osmotic pressure, high temperature, low nutrition capacity and fluctuating processes). Therefore, the stress-induced property of the SED1 gene should be favorable for the cell-surface display of cellulolytic enzymes in CBP. On the other hand, the transcriptional levels of BGL1 controlled by the SED1 promoter decreased dramatically after $48 \mathrm{~h}$ cultivation (Figure 2A). This result suggested that any factors other than stress regulate the expression of SED1 promoter. Identification of the regulatory factors in the expression of SED1 promoter would be necessary for further enhancement of the cellulolytic activities on the cell surface provided using the SS cassette.

In the direct ethanol production from hydrothermally processed rice straw, the BY-EG-SS strain enhanced the saccharification and fermentation of the pretreated rice straw in the presence of a commercial cellulase cocktail (Figure 4). EGII was displayed in the cell surface of the BY-EG-SS strain and successfully compensated for cellulolytic activity of the commercial enzymes under stressful conditions. As stated in the Background Section, the cost of commercial enzymes is one of the biggest obstacles to cost-effective bioethanol production from lignocellulosic materials. The result shown in Figure 4 indicates that cellulase-displaying yeast strains using the SS cassette have significant potential for reducing the cost of commercial enzymes and for the costeffective CBP of lignocellulosic biomass.

It has been reported that the synergetic effects of EG, $\mathrm{CBH}$ and BGL are necessary for the effective hydrolyzation of cellulose using cell-surface-displaying yeasts, and that optimization of the ratios of the activities of these enzymes is also important for the hydrolyzation efficiency $[26,41,42]$. The BY-EG-SS strain clearly improved the ethanol productivity from pretreated rice straw, but this strain expressed only EGII (Figure 4). The cellulolytic activity of this strain could increase with the additional display of other enzymes in the future. In addition, we revealed that the combination of the promoter and anchoring domains in the gene cassette has significant effects on the efficiency of enzyme display. If the activity of various enzymes on the cell surface can be controlled artificially by the combinations of their promoters and anchoring domains, we will be able to construct the recombinant yeast strains optimized for the CBP of lignocellulosic materials.

\section{Conclusions}

We have developed novel gene cassettes for the efficient cell-surface display of exo- and endo-type cellulolytic enzymes. We demonstrated that the combinations of a promoter and an anchoring domain in the display cassette had significant effects on the efficiency with which 
the enzyme integrated into the cell wall. Simultaneous utilization of the SED1 promoter and Sed1 anchoring domain in a gene cassette enabled highly efficient enzyme integration into the cell wall. The BGL-displaying yeast strain carrying this cassette achieved high BGL activity within a relatively short cultivation time. Furthermore, this novel display cassette is also suitable for the hydrolyzation of insoluble substrates including lignocellulosic biomass. Ethanol productivity from hydrothermally processed rice straw was clearly improved by using the novel gene cassettes for EGII display. If cell-surface cellulolytic enzyme activities can be controlled and optimized artificially by the combinations of the promoter and anchoring domain, then cellulasedisplaying yeasts will be an attractive option for cost-effective CBP of lignocellulosic materials.

\section{Methods}

\section{Strains and media}

The host for recombinant DNA manipulation was the Escherichia coli (E. coli) strain NovaBlue (Novagen, Madison, WI, USA). E. coli transformants were grown in Luria-Bertani medium (10 g/L of tryptone, $5 \mathrm{~g} / \mathrm{L}$ of yeast extract and $5 \mathrm{~g} / \mathrm{L}$ of sodium chloride) supplemented with $100 \mu \mathrm{g} / \mathrm{ml}$ of ampicillin at $37^{\circ} \mathrm{C}$. The genetic properties of all yeast strains used in this study are summarized in Table 2. Cell-surface display cassettes of cellulase were expressed in the haploid yeast strain S. cerevisiae BY4741 (LifeTechnologies, Carlsbad, CA, USA). The yeast transformants were screened and pre-cultured in synthetic dextrose (SD) medium $(6.7 \mathrm{~g} / \mathrm{L}$ of yeast nitrogen base without amino acids (Difco Laboratories, Detroit, MI, USA) and $20 \mathrm{~g} / \mathrm{L}$ of glucose) supplemented with appropriate amino acids and nucleic acids in a shaker incubator $(180 \mathrm{rpm})$ at $30^{\circ} \mathrm{C}$, and then aerobically cultured in yeast extract peptone dextrose (YPD) medium (10 g/L of yeast extract, $20 \mathrm{~g} / \mathrm{L}$ of Bactopeptone (Difco Laboratories) and $20 \mathrm{~g} / \mathrm{L}$ of glucose) in a shaker incubator $(150 \mathrm{rpm})$ at $30^{\circ} \mathrm{C}$. Yeast cells were harvested by centrifugation at $1,000 \times \mathrm{g}$ for 5 minutes, then washed twice with distilled water followed by centrifugation at $1,000 \times \mathrm{g}$ for 5 minutes. Wet cell-weight of the washed yeast cells was determined by weighing the cell pellet. The estimated dry cell-weight for all strains was approximately 0.15 -fold the wet cell-weight. The cell pellets were used for the enzyme assays and quantification of transcriptional level as described below.

\section{Plasmid construction and yeast transformation}

The plasmids and primers used in this study are summarized in Tables 1 and 4, respectively. The 3' half of SAG1 cording region (963 bp) and the full length of SED1 cording region except for the start codon $(1,014$ bp) were used as SAG1 and SED1 anchoring regions,
Table 4 PCR primers used in this study

\begin{tabular}{cc}
\hline Primers & Sequence \\
\hline BGL1-F & atgcaactgttcaatttgcc \\
BGL1-PG-R & gggcccgggcccgggttgcaccttcgggagc \\
SAG1a-PG-F & cccgggcccgggcccagcgccaaaagctctt \\
SAG1a-R & taaaatctgcggtgagacgg \\
SED1a-PG-F & cccgggcccgggcccaaattatcaactgtcctattatctgc \\
SED1a-BsrGI-R & gccatctgtacattataagaataacatagcaacaccag \\
SED1a-Xhol-F & gccatcctcgagtaaattatcaactgtcctattatctgc \\
EGII-Ncol-F & gccatcccatgggtcagcagactgtctggggc \\
EGII-Xhol-R & gccatcctcgagccctttcttgcgagacacgag \\
SED1p-CBA-F & cctcttcgctattacgccagattggatatagaaaattaacgtaaggc \\
SED1p-CBA-R & ggcaaattgaacagttgcatcttaatagagcgaacgtatttt \\
VS-CBA-F & aatacgttcgctctattaagatgcaactgttcaatttgcc \\
VS-CBA-R & gttaattttctatatccaatctggcgtaatagcgaagagg \\
\hline
\end{tabular}

respectively. The integrative plasmids for cell-surface display with $T D H 3$ promoter were constructed as follows: the DNA fragment encoding $A$. aculeatus BGL1 was amplified from plasmid pIBG13 [43] by PCR using the BGL1-F and BGL1-PG-R primers. Similarly, the DNA fragments encoding the anchoring domains of $S$. cerevisiae Sag1 and Sed1 were amplified from S. cerevisiae BY4741 [44] genomic DNA by PCR using the SAG1a-PG-F and SAG1a-R primers and SED1a-PG-F and SED1a-BsrGI-R primers, respectively. These anchor fragments were connected to the $3^{\prime}$-terminus of the BGL1 fragment by the fusion PCR method [45] using the BGL1-F and SAG1a-R primers and BGL1-F and SED1a-BsrGI-R primers, respectively. These fragments encoding BGL1-Sag1 and BGL1-Sed1 were digested with $\mathrm{NcoI}$ and $\mathrm{BsrGI}$, and then subcloned into the NcoI and BsrGI sites of pIBG13. The resulting plasmids were named pIBG-TA and pIBG-TS, respectively. The XhoI$B s r G I$ DNA fragment encoding the Sed1 anchoring domain was amplified from S. cerevisiae BY4741 genomic DNA by PCR using the SED1a-XhoI-F and SED1aBsrGI-R primers. This fragment was subcloned into the $X h o \mathrm{I}$ and $B s r \mathrm{GI}$ sites of pIBG13. The resulting plasmid was named pIBG13S. The NcoI-XhoI DNA fragment encoding $T$. reeseii EGII was amplified from p $\delta$ U-PGAGEG [26] by PCR using the EGII-NcoI-F and EGII-XhoI-R primers. This fragment was subcloned into the $\mathrm{NcoI}$ and XhoI sites of pIBG13 and pIBG13S. The resulting plasmids were named pIEG-TA and PIEG-TS, respectively.

The integrative plasmids with the SED1 promoter were constructed as follows: The DNA fragment encoding the SED1 promoter region was amplified from S. cerevisiae BY4741 genomic DNA by PCR using the SED1p-CBA-F and SED1p-CBA-R primers. Likewise, the vector fragment was amplified from pIBG13 by PCR using the VS- 
CBA-F and VS-CBA-R primers. These fragments were connected using the isothermal assembly method [46]. The resulting plasmid was named pISBG13. The NcoIBsrGI DNA fragments encoding BGL1-Sag1, BGL1-Sed1, EGII-Sag1 and EGII-Sed1 were obtained from plasmids pIBG-TA, pIBG-TS, pIEG-TA and pIEG-TS, respectively. Then, these fragments were subcloned into the NcoI and BsrGI site of pISBG13. The resulting plasmids were named pIBG-SA, pIBG-SS, pIEG-SA and pIEG-SS, respectively.

Each plasmid was digested with NdeI within the HIS3 gene. The linearized plasmids were then transformed into S. cerevisiae BY4741 using the lithium acetate method [47], and integrated into the HIS3 locus of the chromosomal DNA by homologous recombination, respectively.

\section{Enzyme assays}

Enzyme activities of the yeast cells were evaluated as described below. BGL activity was assayed with $p$ NPG as the substrate. Washed yeast cells were resuspended in water ( $1 \mathrm{~g}$ wet cells/L): $100 \mu \mathrm{L}$ of the suspended cell or culture supernatant was added to $400 \mu \mathrm{L}$ of $2 \mathrm{mM}$ $p$ NPG solution dissolved in $50 \mathrm{mM}$ sodium citrate buffer, $\mathrm{pH}$ 5.0. After incubation for 10 minutes in a shaker incubator $(500 \mathrm{rpm})$ at $30^{\circ} \mathrm{C}, 500 \mu \mathrm{L}$ of $3.0 \mathrm{M}$ sodium carbonate solution was added to terminate the reaction, and the $p$-nitrophenol liberated was determined based on the absorbance at $400 \mathrm{~nm}$. For these conditions, the molar extinction coefficient of $18,720 \mathrm{M}^{-1} \mathrm{~cm}^{-1}$ was used to calculate the concentration of $p$-nitrophenol in the assay mixture. One unit of enzyme activity was defined as the amount of enzymes required to liberate $1 \mu \mathrm{mol}$ of $p$-nitrophenol per minute.

EG activity was assayed with AZCL-HE-Cellulose, which is a purified insoluble polysaccharide as the substrate. The substrate is supplied commercially in a tablet form as Cellazyme $\mathrm{C}$ tablets (Megazyme, Bray, Ireland). Washed yeast cells were resuspended in water (50 g wet cells/L), then $500 \mu \mathrm{L}$ of the cell suspension was added to $2 \mathrm{~mL}$ of assay solution containing a Cellazyme $\mathrm{C}$ tablet and $50 \mathrm{mM}$ sodium citrate buffer, $\mathrm{pH}$ 5.0. After incubation for $4 \mathrm{~h}$ at $38^{\circ} \mathrm{C}$, endo- $\beta$-1,4-glucanase activity was evaluated based on the absorbance at $590 \mathrm{~nm}$ of the blue dye released into the supernatant.

\section{Quantification of the transcriptional level of the $B G L 1$ gene by real-time PCR}

The transcriptional level was quantified by real-time quantitative-PCR as described previously [48]. The PCR primers BGL 761F and BGL 858R [26] were used for quantification of transcriptional level of BGL1. ACT1 was used as the internal standard.
Direct ethanol production from hydrothermally processed rice straw

Hydrothermally processed rice straw purchased from Mitsubishi Heavy Industries, Ltd. (Tokyo, Japan) was used as a cellulosic material. Ethanol fermentation of the cellulosic material was performed in a $50 \mathrm{~mL}$ polypropylene tube (Corning Inc., Corning, NY, USA) and a heat block (Thermo Block Rotator SN-06BN; Nissin, Tokyo, Japan) as described previously [49]. S. cerevisiae strains used for fermentation were grown under aerobic conditions at $30^{\circ} \mathrm{C}$ for $48 \mathrm{~h}$ in $500 \mathrm{~mL}$ YPD medium. The yeast cells were collected by centrifugation at $1000 \times \mathrm{g}$ for 10 minutes at $20^{\circ} \mathrm{C}$, then washed twice with distilled water. The cells were then resuspended in $10 \mathrm{~mL}$ yeast extract peptone (YP) medium $(10 \mathrm{~g} / \mathrm{L}$ of yeast extract and $20 \mathrm{~g} / \mathrm{L}$ of Bacto-peptone) containing $50 \mathrm{mM}$ sodium citrate buffer (pH 5.0), $100 \mathrm{~g} / \mathrm{L}$ of cellulosic material and 1 FPU/g-biomass of commercial cellulase (Cellic CTec2; Novozymes Inc., Bagsvaerd, Denmark) at an initial cell concentration of $100 \mathrm{~g}$ wet cells/L. Fermentation was initiated by the addition of yeast cells into the tube followed by axial rotation at $35 \mathrm{rpm}$ at $38^{\circ} \mathrm{C}$. The ethanol concentration in the fermentation medium was determined by HPLC (Shimadzu, Kyoto, Japan), as described previously [50].

\section{Additional files}

\begin{abstract}
Additional file 1: Figure S1. Construction of novel gene cassettes with the S. cerevisiae CWP2 promoter and/or its anchoring region. The novel gene cassettes with the S. cerevisiae CWP2 promoter and/or its anchoring region were constructed based on the SS cassette for the yeast cellsurface display of $\beta$-glucosidase (BGL1). All cassettes have the secretion signal sequence of the $R$. oryzae glucoamylase gene and SAG1 terminator

Additional file 2: Figure S2. Time course of $\beta$-glucosidase (BGL) activities of BGL-displaying strains (BY-BG-SC, CS and CC). Recombinant strains harboring gene cassettes shown in Additional file 1: Figure S1 were cultured under aerobic conditions at $30^{\circ} \mathrm{C}$ for $96 \mathrm{~h}$. The culture broth was sampled every $24 \mathrm{~h}$, and BGL activity in the cell was investigated as described in the Methods. Error bars indicate the standard deviations of three independent experiments.
\end{abstract}

\section{Abbreviations}

AZCL-HE-Cellulose: azurine cross-linked hydroxyethylcellulose; BGL: $\beta$ glucosidase; bp: base pairs; $\mathrm{CBH}$ : cellobiohydrolase; CBP: consolidated bioprocessing; EG: endoglucanase; ER: endoplasmic reticulum; FPU: filter paper unit; GPI: glycosylphosphatidylinositol; $p N P G$ : $p$-nitrophenyl- $\beta-D$ glucopyranoside; SA: SED1 promoter and SAG1 anchoring region; SS: SED1 promoter and SED1 anchoring region; TA: TDH3 promoter and SAG1 anchoring region; TS: TDH3 promoter and SED1 anchoring region.

\section{Competing interests}

The authors declare that they have no competing interests.

\section{Authors' contributions}

$\mathrm{Kl}$ designed and performed the experiments, and wrote the manuscript. $\mathrm{TH}$ helped to draft the manuscript. TH and AK supervised the research. All authors read and approved the final manuscript.

\section{Acknowledgements}

We thank Mr Takanobu Yoshida for his assistance and helpful conversations This work was supported by project P07015 of the New Energy and 
Industrial Technology Development Organization (NEDO) under the sponsorship of the Ministry of Economy, Japan.

\section{Author details}

'Department of Chemical Science and Engineering, Graduate School of Engineering, Kobe University, 1-1 Rokkodai, Nada, Kobe 657-8501, Japan. ${ }^{2}$ Organization of Advanced Science and Technology, Kobe University, 1-1 Rokkodai, Nada, Kobe 657-8501, Japan. ${ }^{3}$ Biomass Engineering Program, RIKEN, 1-7-22 Suehiro-cho, Tsurumi-ku, Yokohama, Kanagawa 230-0045, Japan. ${ }^{4}$ Department of Food Bioscience and Technology, College of Life Sciences and Biotechnology, Korea University, Seoul 136-713, Republic of Korea.

Received: 23 August 2013 Accepted: 24 December 2013

Published: 14 January 2014

\section{References}

1. Hasunuma T, Kondo A: Development of yeast cell factories for consolidated bioprocessing of lignocellulose to bioethanol through cell surface engineering. Biotechnol Adv 2012, 30:1207-1218.

2. Alvira P, Tomás-Pejó E, Ballesteros M, Negro MJ: Pretreatment technologies for an efficient bioethanol production process based on enzymatic hydrolysis: a review. Bioresour Technol 2010, 101:4851-4861.

3. da Costa Sousa L, Chundawat SP, Balan V, Dale BE: 'Cradle-to-grave' assessment of existing lignocellulose pretreatment technologies. Curr Opin Biotechnol 2009, 20:339-347.

4. Hendriks AT, Zeeman G: Pretreatments to enhance the digestibility of lignocellulosic biomass. Bioresour Technol 2009, 100:10-18.

5. Percival Zhang YH, Himmel ME, Mielenz JR: Outlook for cellulase improvement: screening and selection strategies. Biotechnol Adv 2006 , 24:452-481.

6. Xu Q, Singh A, Himmel ME: Perspectives and new directions for the production of bioethanol using consolidated bioprocessing of lignocellulose. Curr Opin Biotechnol 2009, 20:364-371.

7. Lynd LR, van Zyl WH, McBride JE, Laser M: Consolidated bioprocessing of cellulosic biomass: an update. Curr Opin Biotechnol 2005, 16:577-583.

8. Cardona CA, Sánchez OJ: Fuel ethanol production: process design trends and integration opportunities. Bioresour Technol 2007, 98:2415-2457.

9. Pandey A: Handbook of plant-based biofuels. Boca Raton: CRC Press; 2009.

10. Olsson L, Hähnhagerdal B: Fermentative Performance of Bacteria and Yeasts in Lignocellulose Hydrolysates. Process Biochem 1993, 28:249-257.

11. Lau MW, Gunawan C, Balan V, Dale BE: Comparing the fermentation performance of Escherichia coli KO11, Saccharomyces cerevisiae 424A (LNH-ST) and Zymomonas mobilis AX101 for cellulosic ethanol production. Biotechnol Biofuels 2010, 3:11.

12. van Zyl WH, Lynd LR, den Haan R, McBride JE: Consolidated bioprocessing for bioethanol production using Saccharomyces cerevisiae. Biofuels 2007, 108:205-235.

13. Fujita Y, Ito J, Ueda M, Fukuda H, Kondo A: Synergistic saccharification, and direct fermentation to ethanol, of amorphous cellulose by use of an engineered yeast strain codisplaying three types of cellulolytic enzyme. Appl Environ Microbiol 2004, 70:1207-1212.

14. Fujita Y, Takahashi S, Ueda M, Tanaka A, Okada H, Morikawa Y, Kawaguchi T, Arai M, Fukuda H, Kondo A: Direct and efficient production of ethanol from cellulosic material with a yeast strain displaying cellulolytic enzymes. Appl Environ Microbiol 2002, 68:5136-5141.

15. Kondo A, Ueda M: Yeast cell-surface display-applications of molecular display. Appl Microbiol Biotechnol 2004, 64:28-40.

16. Kotaka A, Bando H, Kaya M, Kato-Murai M, Kuroda K, Sahara H, Hata Y, Kondo A, Ueda M: Direct ethanol production from barley $\beta$-glucan by sake yeast displaying Aspergillus oryzae $\beta$-glucosidase and endoglucanase. J Biosci Bioeng 2008, 105:622-627.

17. Yamada R, Taniguchi N, Tanaka T, Ogino C, Fukuda H, Kondo A: Direct ethanol production from cellulosic materials using a diploid strain of Saccharomyces cerevisiae with optimized cellulase expression. Biotechnol Biofuels 2011, 4:8.

18. Yanase S, Yamada R, Kaneko S, Noda H, Hasunuma T, Tanaka T, Ogino C, Fukuda $\mathrm{H}$, Kondo A: Ethanol production from cellulosic materials using cellulase-expressing yeast. Biotechnol J 2010, 5:449-455.

19. Ueda M, Tanaka A: Cell surface engineering of yeast: construction of arming yeast with biocatalyst. J Biosci Bioeng 2000, 90:125-136.
20. Kondo A, Shigechi H, Abe M, Uyama K, Matsumoto T, Takahashi S, Ueda M, Tanaka A, Kishimoto M, Fukuda H: High-level ethanol production from starch by a flocculent Saccharomyces cerevisiae strain displaying cell-surface glucoamylase. Appl Microbiol Biotechnol 2002, 58:291-296.

21. Matano Y, Hasunuma T, Kondo A: Simultaneous improvement of saccharification and ethanol production from crystalline cellulose by alleviation of irreversible adsorption of cellulase with a cell surfaceengineered yeast strain. Appl Microbiol Biotechnol 2013, 97:2231-2237.

22. Shimoi $H$, Kitagaki $H$, Ohmori $H$, limura $Y$, Ito $K$ : Sed1 $p$ is a major cell wall protein of Saccharomyces cerevisiae in the stationary phase and is involved in lytic enzyme resistance. J Bacteriol 1998, 180:3381-3387.

23. Jones ME: Analysis of algebraic weighted least-squares estimators for enzyme parameters. Biochem J 1992, 288(Pt 2):533-538.

24. Ten LN, Im WT, Kim MK, Kang MS, Lee ST: Development of a plate technique for screening of polysaccharide-degrading microorganisms by using a mixture of insoluble chromogenic substrates. J Microbiol Methods 2004, 56:375-382.

25. van der Vaart JM, te Biesebeke R, Chapman JW, Toschka HY, Klis FM, Verrips CT: Comparison of cell wall proteins of Saccharomyces cerevisiae as anchors for cell surface expression of heterologous proteins. App/ Environ Microbiol 1997, 63:615-620.

26. Yamada R, Taniguchi N, Tanaka T, Ogino C, Fukuda H, Kondo A: Cocktail $\delta$ integration: a novel method to construct cellulolytic enzyme expression ratio-optimized yeast strains. Microb Cell Fact 2010, 9:32.

27. Decker $\mathrm{CH}$, Visser J, Schreier P: $\beta$-glucosidases from five black Aspergillus species: study of their physico-chemical and biocatalytic properties. $J$ Agric Food Chem 2000, 48:4929-4936.

28. Spellman PT, Sherlock G, Zhang MQ, lyer VR, Anders K, Eisen MB, Brown PO, Botstein D, Futcher B: Comprehensive identification of cell cycleregulated genes of the yeast Saccharomyces cerevisiae by microarray hybridization. Mol Biol Cell 1998, 9:3273-3297.

29. Pittet M, Conzelmann A: Biosynthesis and function of GPI proteins in the yeast Saccharomyces cerevisiae. Biochim Biophys Acta 2007, 1771:405-420.

30. Caro LH, Tettelin H, Vossen JH, Ram AF, van den Ende H, Klis FM: In silicio identification of glycosyl-phosphatidylinositol-anchored plasmamembrane and cell wall proteins of Saccharomyces cerevisiae. Yeast 1997, 13:1477-1489.

31. Vossen JH, Müller WH, Lipke PN, Klis FM: Restrictive glycosylphosphatidylinositol anchor synthesis in cwh6/gpi3 yeast cells causes aberrant biogenesis of cell wall proteins. J Bacteriol 1997, 179:2202-2209.

32. Hamada K, Fukuchi S, Arisawa M, Baba M, Kitada K: Screening for glycosylphosphatidylinositol (GPI)-dependent cell wall proteins in Saccharomyces cerevisiae. Mol Gen Genet 1998, 258:53-59.

33. Hamada K, Terashima H, Arisawa M, Yabuki N, Kitada K: Amino acid residues in the $\omega$-minus region participate in cellular localization of yeast glycosylphosphatidylinositol-attached proteins. J Bacteriol 1999, 181:3886-3889.

34. Frieman MB, Cormack BP: The $\omega$-site sequence of glycosylphosphatidylinositol-anchored proteins in Saccharomyces cerevisiae can determine distribution between the membrane and the cell wall. Mol Microbiol 2003, 50:883-896.

35. van der Vaart JM, Caro LH, Chapman JW, Klis FM, Verrips CT: Identification of three mannoproteins in the cell wall of Saccharomyces cerevisiae. J Bacteriol 1995, 177:3104-3110.

36. Doering TL, Schekman R: GPI anchor attachment is required for Gas $1 p$ transport from the endoplasmic reticulum in COP II vesicles. EMBO J 1996, 15:182-191.

37. Dupres $V$, Dufrêne $Y F$, Heinisch JJ: Measuring cell wall thickness in living yeast cells using single molecular rulers. ACS Nano 2010, 4:5498-5504.

38. Schreuder MP, Mooren AT, Toschka HY, Verrips CT, Klis FM: Immobilizing proteins on the surface of yeast cells. Trends Biotechnol 1996, 14:115-120.

39. Shimojyo R, Furukawa $H$, Fukuda $H$, Kondo A: Preparation of yeast strains displaying lgG binding domain ZZ and enhanced green fluorescent protein for novel antigen detection systems. J Biosci Bioeng 2003, 96:493-495.

40. Wu H, Zheng X, Araki Y, Sahara H, Takagi H, Shimoi H: Global gene expression analysis of yeast cells during sake brewing. Appl Environ Microbiol 2006, 72:7353-7358.

41. Dashtban M, Schraft H, Qin W: Fungal bioconversion of lignocellulosic residues; opportunities \& perspectives. Int J Biol Sci 2009, 5:578-595. 
42. Stricker $A R$, Mach RL, de Graaff $L H$ : Regulation of transcription of cellulases- and hemicellulases-encoding genes in Aspergillus niger and Hypocrea jecorina (Trichoderma reesei). Appl Microbiol Biotechnol 2008, 78:211-220.

43. Katahira S, Mizuike A, Fukuda H, Kondo A: Ethanol fermentation from lignocellulosic hydrolysate by a recombinant xylose- and cellooligosaccharide-assimilating yeast strain. Appl Microbiol Biotechnol 2006, 72:1136-1143.

44. Brachmann CB, Davies A, Cost GJ, Caputo E, Li J, Hieter P, Boeke JD: Designer deletion strains derived from Saccharomyces cerevisiae S288C: a useful set of strains and plasmids for PCR-mediated gene disruption and other applications. Yeast 1998, 14:115-132.

45. Cha-aim K, Fukunaga T, Hoshida H, Akada R: Reliable fusion PCR mediated by GC-rich overlap sequences. Gene 2009, 434:43-49.

46. Gibson DG, Young L, Chuang RY, Venter JC, Hutchison CA 3rd, Smith HO: Enzymatic assembly of DNA molecules up to several hundred kilobases. Nat Methods 2009, 6:343-345.

47. Chen DC, Yang BC, Kuo TT: One-step transformation of yeast in stationary phase. Curr Genet 1992, 21:83-84

48. Ismail KS, Sakamoto T, Hatanaka H, Hasunuma T, Kondo A: Gene expression cross-profiling in genetically modified industrial Saccharomyces cerevisiae strains during high-temperature ethanol production from xylose. J Biotechnol 2013, 163:50-60.

49. Matano $Y$, Hasunuma T, Kondo A: Display of cellulases on the cell surface of Saccharomyces cerevisiae for high yield ethanol production from high-solid lignocellulosic biomass. Bioresour Technol 2012, 108:128-133.

50. Hasunuma T, Sung K, Sanda T, Yoshimura K, Matsuda F, Kondo A: Efficient fermentation of xylose to ethanol at high formic acid concentrations by metabolically engineered Saccharomyces cerevisiae. Appl Microbiol Biotechnol 2011, 90:997-1004

doi:10.1186/1754-6834-7-8

Cite this article as: Inokuma et al: Efficient yeast cell-surface display of exo- and endo-cellulase using the SED1 anchoring region and its original promoter. Biotechnology for Biofuels 2014 7:8.

\section{Submit your next manuscript to BioMed Central and take full advantage of:}

- Convenient online submission

- Thorough peer review

- No space constraints or color figure charges

- Immediate publication on acceptance

- Inclusion in PubMed, CAS, Scopus and Google Scholar

- Research which is freely available for redistribution 